\title{
Narrativas digitales para potenciar la creatividad, la participación y el pensamiento crítico en la enseñanza obligatoria
}

\section{Digital narratives to improve creativity, participation and critical thinking at compulsory education level}

\author{
Roi Guitián, María Lobo, Sonia Díaz \\ epd@agareso.es, participacion@agareso.es, organizacion@agareso.es \\ Educación para el Cambio Social \\ AGARESO \\ Santiago de Compostela, España
}

\begin{abstract}
Resumen- Memes, gif, fotografías o vídeos de Youtube forman un ecosistema mediático con sus propias reglas, un nuevo código lingüístico con el que nos comunicamos y con el que, por lo tanto, aprendemos. ¿Cómo afecta a nuestro imaginario sociocultural? ¿Estamos preparando al alumnado para comprender y para utilizar estos recursos desde la enseñanza obligatoria? ¿Cómo fomentar la creatividad, la participación ciudadana y el pensamiento crítico a través de esta narrativa digital? EPDLAB es un proyecto que se ha realizado en Galicia durante los últimos 4 cursos escolares. A través del análisis del lenguaje mediático y de la reflexión colectiva, diferentes grupos de estudiantes de ESO elaboran contenidos digitales alrededor de temas como la igualdad, la inclusión o la justicia social. Un proyecto abierto y participativo que defiende una educación comprometida con la comunidad en la que vive.
\end{abstract}

Palabras clave: Educación en valores, Alfabetización mediática, Pensamiento crítico, Acción y transformación social, Participación

Abstract-Memes, gif, photos or Youtube videos are part of our media enviroment. A new enviroment with its own rules. A new linguistic code to communicate and to learn. How does that impact on our social and cultural imaginary? Are we training students to understand and use these means at compulsory education level? How could we use theses digital narratives to improve creativity, citizen participation and critical thinking? EPDLAB is a project that has been carried out in Galicia during last 4 school years. Different groups of secundary students produce digital contents on subjects such as equality, inclusion or social justice from relevant collective discussion and media language analysis. An open and participatory project that defend an education committed to the community it takes place in.

Keywords: Values Education, Media Literacy, Critical Thinking, Social Action and Transformation, Citizen Participation

\section{INTRODUCCIÓN}

EPDLAB es un proyecto que se desarrolla en el ámbito de la educación formal, a partir del concepto de "folclore digital", que explora ampliamente Jaron Rowan (2015) y que hace referencia a los contenidos creados en de forma amateur y reinventados una $\mathrm{y}$ otra vez por personas anónimas.
Incorporamos estas creaciones espontáneas propias del entorno de Internet como herramientas de comunicación.

Una de las características del folclore es que, vinculado a la tradición oral y a los saberes compartidos, la noción de autoría se diluye y una creación concreta puede ser reinterpretada y adaptada sin limitaciones, ya que pasa a formar parte de una comunidad concreta como propietaria colectiva de esa pieza. Desde esta noción de narraciones colectivas y populares, con un valor concreto para la comunidad, es desde donde entendemos que se debe trabajar la educomunicación: a partir del diálogo y desde una perspectiva constructiva. Porque, enunciando alguna de las ideas claves de "la educación expandida", tal y como la aborda el colectivo Zemos98, la educación puede suceder en cualquier momento y en cualquier lugar; la educación no es, está siendo.

Recuperamos también una idea indispensable: la educación para la participación activa, el talón de Aquiles en muchos procesos colaborativos. En general, la sensación es que aprendemos a escuchar y a recibir, pero no aprendemos a gestionar la participación y la construcción de soluciones colectivas. ¿Puede ser que aprendamos a consumir pero que no aprendamos a crear?

La cultura contemporánea se construye, además, en espacios tanto físicos como virtuales, entendiendo además que los medios de comunicación son un soporte para la transmisión y el aprendizaje. Y entendemos también que las redes sociales son medios de comunicación. En este sentido, el proyecto trabaja sobre la representación de los diferentes colectivos en los relatos mediáticos y reflexiona sobre cómo estos construyen estereotipos que se convierten en realidades y actitudes. $\mathrm{O}$, al menos, en nuestra forma de conocer e interactuar con otras realidades.

Del mismo modo que el proyecto se apoya en las teorías sobre convergencias culturales desarrolladas especialmente por Henry Jenkins (2006), que hablan de la capacidad de los discursos populares como forma de interacción y confrontación con los de los medios tradicionales. Las teorías de Jenkins exponen que la inteligencia colectiva puede entenderse como una alternativa al poder mediático y que estamos aprendiendo a 
usar ese poder a diario a través de nuestras interacciones dentro de una "cultura convergente".

Porque todo sujeto es, en definitiva, un sujeto cultural. Sin embargo, la cultura no es algo estático y aislado en un marco especializado, la cultura es algo vivo, que se comparte, se transmite y se aprende. Y, por tanto, también cambia cuando cambian las comunidades que la sostienen. En este contexto, se hace inevitable incorporar la enseñanza de habilidades para buscar, leer, comprender y aprehender de forma autónoma y significativa, como un paso necesario para construir una ciudadanía activa, solidaria y responsable.

\section{CONTEXTO}

EPDLAB es un proyecto de la Organización No Gubernamental para el Desarrollo (ONGD) AGARESO, que cuenta con la financiación de la convocatoria de Educación para o Desenvolvemento e a Cidadanía Global de Cooperación Galega y que se viene llevando a cabo en centros de secundaria de Galicia ininterrumpidamente desde 2015.

La LOMCE reconoce, entre sus siete competencias clave, la competencia digital, que es aquella que "implica el uso creativo, crítico y seguro de las tecnologías de la información y la comunicación para alcanzar los objetivos relacionados con el trabajo, la empleabilidad, el aprendizaje, el uso del tiempo libre, la inclusión y participación en la sociedad."

Asimismo, sabemos que nuestro conocimiento de la realidad está mediado. Incluso algunas realidades que forman parte de nuestra propia comunidad resultan invisibles, y solo aparecen en el discurso público como relatos unidireccionales construidos para crear opinión. La educación obligatoria enuncia en su preámbulo que "el aprendizaje en la escuela debe ir dirigido a formar personas autónomas, críticas, con pensamiento propio". Así pues, el contexto social y educativo demanda poner el foco sobre aquellos aprendizajes que construyen autonomía y pensamiento crítico, pero ¿cómo?

EPDLAB propone trabajar ese pensamiento crítico, específicamente relacionado con la educación en valores centrada en el desarrollo y la justicia social, desde una parcela muy concreta, como son la capacidad de lectura crítica de medios, el análisis y la comprensión de los discursos en función de emisores, receptores, soportes y contexto. Ni la tecnología ni las narrativas asociadas a ella son neutrales. Y debemos visibilizar esa falta de neutralidad, con el objetivo de despertar interés $\mathrm{y}$, también, de experimentar con ellas. Análisis y creación son inherentes a cualquier proceso de aprendizaje.

Los cinco centros educativos gallegos que participan cada curso escolar en EPDLAB son escogidos en función de dos parámetros: que representen realidades socioeconómicas y culturales diferentes, para que el alumnado sea consciente de la diversidad de su entorno más próximo y de el valor de dicha diversidad en su proceso de aprendizaje, y que el profesorado y el equipo directivo esté implicado en el proyecto, lo que garantiza su funcionamiento, favorece la continuidad y permite la imbricación con el proyecto de centro. En cada uno de ellos, hay un profesor o profesora de referencia, que ya ha participado o coordinado actividades de educación para la transformación social y/o en el uso de las TIC (tecnologias de la información y la comunicación) en el aula.

Además de los centros gallegos, cada año participa puntualmente en el proyecto una escuela centroamericana, con la que la ONGD está trabajando en alguno de sus proyectos de Cooperación Internacional, con el objetivo de que el estudiantado comparta sus problemáticas e inquietudes (falta de oportunidades, aislamiento, escasa oferta cultural y de ocio, etc.) y asimile cuestiones como las habilidades interculturales y la representación alternativa al discurso etnocéntrico, desde el aprendizaje activo y la comunicación interpersonal.

En un contexto donde prima el consumo pasivo y acrítico de contenidos, es necesario trabajar en la lectura y la producción de discursos relevantes. En la educación obligatoria, esto es parte del trabajo con alumnado y profesorado. Por eso, EPDLAB contó desde el principio con docentes que, desde varios centros, acompañan a AGARESO en los procesos, aportan mejoras e introducen adaptaciones. Buscando la formación continua y la replicabilidad de cada una de las acciones, estos procesos se comparten en redes sociales (por parte de alumnado), en un blog (profesorado) y en forma de guía didáctica bajo licencia Reconocimiento - No Comercial Compartir Igual (CC-BY-SA) por parte del personal técnico de la ONGD, con el objetivo de que los aprendizajes se expandan $\mathrm{y}$ sean múltiples, generadores de nuevos espacios de aprendizaje, de forma que el conjunto de la comunidad educativa se beneficie de cada resultado.

\section{DESCRIPCIÓN}

\section{Metodologías}

EPDLAB es un proyecto de educomunicación, que entiende la acción educativa como algo indisociable de la acción comunicativa. A nivel teórico, tiene su base en el trabajo de Mario Kaplún, un comunicador que, a su vez, bebe de las teorías educativas del aprendizaje como una construcción cultural de Lev Vigotsky, en el modelo de escuela popular desarrollado entorno a las teorías de Celèstin Freinet, o la perspectiva que aportó la pedagogía del oprimido de Paulo Freire, entre otros.

Se trata, por tanto, de un proyecto diseñado siguiendo un doble enfoque pedagógico: el constructivismo y el aprendizaje entre iguales o red entre pares (P2P, peer-to-peer) Partimos de una perspectiva constructivista, en la que la experiencia propia de cada persona será el elemento clave para la construcción de un proceso de aprendizaje propio. De esta manera, combinamos metodologías de trabajo basadas en la observación y en el análisis individual, así como en la reflexión colectiva (debates y dinámicas de puesta en común) y en el trabajo en grupo. El objetivo es promover el aprendizaje entre iguales como dinámica de construcción de conocimiento, así como formar al alumnado en dinámicas sociales que fomenten el diálogo, la colaboración y la innovación en la busca de soluciones compartidas. Todos los aprendizajes en EPDLAB tienen un enfoque multidireccional, activo y participativo.

Cada actividad sigue un mismo proceso de aprendizaje: motivación e identificación de ideas previas; introducción de nuevos conceptos y habilidades; aplicación de 
los conceptos y habilidades adquiridas; y revisión y práctica del aprendizaje.

El aprendizaje es significativo, motivando el interés del alumnado mediante vínculos con su realidad cotidiana, su participación relevante y su pensamiento crítico. Por lo tanto, en cada una de las sesiones, se trabaja la educación en valores centrada en el desarrollo y la justicia social, a partir de realidades narradas desde los medios de comunicación en relación con las experiencias individuales y grupales del alumnado. Las sesiones parten del análisis de esta realidad, de su relato y de su representación mediática, se desarrollan a través de la reflexión colectiva y el pensamiento crítico, para finalizar con la planificación y realización de un producto multimedia siguiendo la metodología del trabajo colaborativo, en la que el alumnado tendrá que tomar decisiones, argumentar sus aportaciones, buscar el consenso y participar activamente en las diferentes dinámicas grupales.

Durante este proceso reflexivo y creativo, las personas que facilitan las sesiones van introduciendo una mayor complejidad en las tareas de análisis y de producción, destacando los prejuicios y estereotipos que determinan nuestra visión del mundo desde una perspectiva mediática, como individuos y como sociedad. La idea es que aprendan a partir de su experiencia y reflexiones, desde una perspectiva activa $\mathrm{y}$ relevante para sus intereses y necesidades (aprendizaje significativo), así como a partir de metodologías prácticas enfocadas a la solución de problemas, a la creatividad, a la expresividad y a la innovación educativa (aprender haciendo).

Las sesiones son facilitadas por personal de la ONGD especializado en educomunicación, con la finalidad de apoyar el trabajo en equipo y de que las dificultades técnicas no obstaculicen el trabajo sobre competencias y contenidos. Del mismo modo, como actividad que mezcla comunicación y educación, el foco no está en el producto final (que, muchas veces, es mejorable a nivel técnico), sino en el proceso de creación. Tratamos de encontrar un equilibrio entre un resultado visible y compartible en la red con un trabajo relevante para el alumnado. Es preciso tener en cuenta que hay alumnado muy joven y que, además, el proyecto quiere huir de una visión tecnológica o tecnocrática del aprendizaje: la calidad técnica no es lo más relevante, sino la apuesta por una visión más humanista, en la que las herramientas son únicamente eso, herramientas que facilitan un trabajo determinado, en un contexto específico para un público concreto. Y que si alguno de estos tres elementos se modifican la herramienta puede seguir siendo válida aunque el resultado sea diferente.

En consecuencia, nuestro enfoque metodológico no prioriza los aspectos relacionados con la técnica de las producciones, ya que consideramos a las TIC como instrumentos y no como un fin en sí mismo. En esta misma línea, el alumnado no trabaja con equipos de grabación o edición profesionales, ya que demoraría la realización de las actividades, dificultaría la participación de los grupos y no son las herramientas presentes en su día a día. Trabajamos con dispositivos presentes en el aula, sean del centro y/o personales (móviles, tabletas, portátiles), para que puedan conocer su funcionamiento $\mathrm{y}$ expandir su uso más allá del aula y del proyecto. El objetivo último es trabajar tanto habilidades comunicativas (lectura y comprensión de contenidos mediáticos, reconocimiento del discurso y de los géneros, lectura y pensamiento visual $\mathrm{y}$ gráfico, producción de mensajes multimedia, expresividad y argumentación, etc.), como habilidades sociales y culturales (trabajo en grupo, relaciones interculturales, pensamiento global y local, colaboración, solución de problemas, etc.)

Los conocimientos y habilidades se ponen en práctica mediante la creación de productos digitales (multimedia y audiovisuales), alrededor de la educación en valores y el desarrollo, enfocándonos sobre la desigualdad y la justicia social desde una perspectiva mediática, siguiendo el eje temático descrito en los Objetivos de Desarrollo Sostenible de Naciones Unidas: lenguaje inclusivo, representación de colectivos minorizados, interculturalidad, cultura de paz, derechos humanos, desequilibrios Norte-Sur, economías solidarias, comercio justo, igualdad y género... Generando nuevos materiales de educación y sensibilización de carácter innovador, mediante el empleo de las narrativas digitales y los relatos transmedia.

Para conocer y ahondar en el valor de la comunicación como herramienta de cambio y en el papel de la Cooperación como estrategia para conseguir la igualdad, trabajamos según un itinerario didáctico que explora las relaciones entre realidades individuales y realidades colectivas, así como su relevancia en la construcción de una misma sociedad. Este itinerario se desarrolla alrededor de tres grandes bloques temáticos, correspondientes a los tres trimestres del curso escolar, comenzando desde una perspectiva local, a partir de representaciones individuales y/o cercanas, para finalizar con una visión global sobre la desigualdad, su representación mediática y el poder de la comunicación como herramienta de transformación social.

\section{Actividades}

El proyecto EPDLAB se desarrolla a lo largo de un curso escolar en cada uno de los cinco centros participantes, en sesiones mensuales de 100 minutos dentro del horario lectivo. Esto implica que al menos dos docentes han de ceder una de sus horas de clase al mes para el proyecto. Su participación en la sesión ha de ser, además, activa, colaborando en el desarrollo de la actividad y recogiendo posteriormente lo trabajando en el blog del aula, a modo de crónica.

Estas sesiones han ido modificándose a lo largo de los años, atendiendo a las evaluaciones realizadas tanto por las y los docentes como por la evaluadora externa, que cada curso elabora un informe técnico de cumplimiento de objetivos y de propuestas de mejora.

El itinerario, se subdivide en tres grandes bloques:

1. Igualdad e Inclusión social. A lo largo del primer trimestre, trabajamos alrededor de la comunicación inclusiva, entendida como una herramienta para promover la igualdad en el acceso a los recursos y a las oportunidades. Exploramos los relatos mediáticos para analizar la representación de los diferentes grupos sociales, así como la influencia de estos relatos en las relaciones entre los individuos de una sociedad. Comenzamos trabajando con la identidad propia, los roles de género y la 
subjetividad como puntos de partida, con la finalidad de activar emociones como la empatía y la iniciativa personal, que actúan como fuerzas generadoras de cambio.

2. Interculturalidad. En el segundo trimestre analizamos la representación de los diferentes colectivos culturales, y damos un primer paso para hablar de las relaciones Norte-Sur. Nos centramos en el lenguaje empleado por los medios de comunicación para describir y representar las migraciones, las minorías culturales y las relaciones interculturales. Y hablamos de lenguaje multimedia, no únicamente el textual: imágenes, gráficos, vídeos, sonidos, etc. El objetivo es reconocer la importancia de los relatos mediáticos en nuestras relaciones con otros colectivos y culturas, tanto en un sentido positivo como negativo. Trabajamos con bulos o noticias falsas, ampliando el enfoque del anterior bloque desde el ámbito individual o familiar a un ámbito social más extenso.

3. Relaciones Norte-Sur. Después de dos trimestres trabajando en la reflexión sobre nuestra propia realidad, en primer lugar, y en la reflexión sobre nuestra relación con otras culturas, en el segundo, llega el momento de mirar al planeta como una realidad única. Así, en el tercer y último trimestre, trabajamos alrededor de una perspectiva global que nos acerca a las relaciones socioeconómicas que marcan las diferencias entre los países del Norte y los países del Sur, incidiendo en el valor de la cooperación internacional y en la participación activa como formas de incidir en nuestra realidad desde lo local a lo global.

\section{Recursos}

En cada una de las sesiones, abordamos una temática a partir del análisis y la reflexión de su representación en los medios, tanto análogicos como digitales, y producimos una serie de contenidos digitales. ¿Por qué emplear estos recursos digitales en el aula?

Hay un anglicismo que desde hace unos años se emplea con frecuencia: "digital storytelling". A lo largo de nuestra Historia, sabemos que la comunicación verbal servía para transmitir conocimientos, procesos, experiencias... Es decir, para enseñar y para aprender. Las historias siempre han sido una herramienta para el conocimiento y la comunicación, así como un soporte para la cultura colectiva, para el patrimonio inmaterial de una comunidad. Estas historias, además de transmitir conocimientos y saberes, transmiten una forma de entender el mundo, las personas que lo habitan y las relaciones entre ellas.

Sabemos que los mensajes mediáticos son el soporte de nuestro imaginario cultural y, por tanto, determinan nuestra relación con el resto del mundo. $\mathrm{Si}$ estas historias vienen descritas desde la burbuja de filtros de Eli Pariser (2017) y desde un punto de vista etnocéntrico, si estos relatos no son entendidos en un contexto determinado y si sus recursos expresivos no son identificados como tales, tenemos narrativas llenas de estereotipos, prejuicios, racismo y, en general, de desigualdad basada en relaciones de poder. La escuela debe ser el lugar donde aprendamos a leer y comprender estos relatos, a identificar los recursos expresivos y su rol en ellos, a reconocer el contexto y la intencionalidad, de cara a formar una ciudadanía responsable, autónoma y solidaria.
Por otro lado, si estas narrativas (digitales) conforman en gran medida nuestra forma de conocer el mundo y por tanto nuestras acciones en él, ¿no ha llegado el momento de apoderarse de estas herramientas y usarlas en función de nuestras expectativas y necesidades? Para nosotras, la respuesta está clara y, sin caer en el optimismo tecnológico, sí creemos que tienen un gran potencial en el aula de cara a fomentar el pensamiento crítico, la ciudadanía activa y el aprendizaje significativo, ya que conforman un lenguaje que favorece la expresividad, la creatividad y la participación.

El aprendizaje es un proceso que requiere una vinculación emocional: aprehender conocimientos y desarrollar habilidades son acciones que se producen si son procesos vinculados a nuestra realidad, destinados a responder a nuestras expectativas y a cubrir nuestras necesidades. Así, creemos que es imprescindible introducir en las aulas el lenguaje con el que la sociedad se comunica, el código de comunicación del mundo en el que vivimos. Si el modelo comunicativo determina nuestras relaciones y nuestro imaginario cultural, no podemos dejarlo al margen.

Las narrativas digitales llenas de estereotipos y prejuicios proyectan actitudes discriminatorias dentro y fuera de la escuela. El alumnado recibe y comparte estos contenidos en sus redes sociales, un espacio donde se comunican y se relacionan con naturalidad. Trabajar la convivencia en positivo implica educarlos para saber reconocer, participar y tomar decisiones en la red en contra de narrativas que fomenten contravalores, enseñándoles a utilizar herramientas y lenguajes que les son cercanos, como el digital.

Trabajar en la educación formal con micronarrativas -tal y como las describió el proyecto Embed.at para la Universidad Internacional de Andalucía- nos permite también poner el foco en los procesos y no tanto en los resultados. Una idea clave a la hora de utilizar las narrativas digitales o el audiovisual en el aula, que ya citamos previamente, es la de que no podemos centrar los objetivos pedagógicos en la calidad del producto resultante, sino en los valores inherentes al proceso creativo: reflexión, análisis, diálogo grupal y trabajo en equipo, que contribuyen al aprendizaje de la convivencia escolar y al establecimiento de relaciones más positivas construidas desde la diversidad y desde la cooperación

A modo de resumen, y obviando los formatos "clásicos" que en cada sesión utilizamos como herramientas de análisis (prensa escrita, programas de TV, publicidad...), las (micro) narrativas digitales que incorporamos en el trabajo educación formal son: memes, doblajes, vídeos breves (algunos de ellos, musicales, otros en bucle), TikTok, Instagram stories, gif, contrapublicidad, stop-motion... Recursos, todos ellos, que comparten una serie de características: humor, brevedad, autoreferencialidad, remezcla... y que forman parte de ese ecosistema digital al que hicimos referencia al principio, que nos habla de incorporar el folclore digital a las aulas como recurso para el aprendizaje.

\section{Resultados}

Con este proyecto conseguimos que el alumnado aprenda a elaborar sus propios discursos desde una visión reflexiva y crítica de la realidad que nos rodea, haciendo un uso 
responsable de la tecnología, de las herramientas para el intercambio de experiencias y conocimientos, compartiendo y empatizando con la realidad de otros chicos y chicas que viven en otras zonas de la región en otros países del Sur.

Para medir el impacto en el cambio del discurso del propio alumnado, realizamos encuestas antes de comenzar con el proyecto y una evaluadora externa compara los resultados de con las respuestas que ofrece el alumnado y el profesorado al finalizar el curso. También analizamos los trabajos hechos en las formaciones, tanto en el contenido como en la forma. Y también contribuye a esta medición y análisis el propio diario del proyecto, en el que se van registrando los avances y cambios de actitud del propio alumnado, aunque esta vez a nivel cualitativo. Para esto, se utilizan dos herramientas: el blog epdlab.gal/blog y las cuentas de aula en Instagram y Twitter, gestionadas por el profesorado y por alumnado de cada centro.

El impacto en las personas que participan directamente en él es, cada año, óptimo. A modo de ejemplo sobre los resultados documentados por la evaluación externa, incorporamos un gráfico con algunas de las manifestaciones del alumnado cuando se les pide que definan el proyecto con una palabra:

\begin{tabular}{|c|c|c|c|}
\hline \multicolumn{2}{|c|}{$\begin{array}{l}\text { "Algo único que debería estar } \\
\text { presente en todos os centros de } \\
\text { educación" }\end{array}$} & $\begin{array}{l}\text { "Proxecto para } \\
\text { melloraro } \\
\text { mundo" }\end{array}$ & $\begin{array}{l}\text { "Un proxecto polo que } \\
\text { todos deberiamos pasar" }\end{array}$ \\
\hline \multicolumn{2}{|c|}{$\begin{array}{l}\text { "Un proxecto para darnos a coñecer } \\
\text { a realidade e concienciarnos que } \\
\text { todos podemos colaborar" }\end{array}$} & \multicolumn{2}{|c|}{$\begin{array}{c}\text { "O EpDLab axúdanos a poder cambiar o noso } \\
\text { pensamento e é un dos mellores métodos de } \\
\text { aprendizaxe" }\end{array}$} \\
\hline \multicolumn{2}{|c|}{ "O mellor proxecto do mundo" } & & "Unha fase \\
\hline \multicolumn{2}{|c|}{$\begin{array}{l}\text { "Aprender cousas que non } \\
\text { sabiamos do mundo" }\end{array}$} & & $\min "$ \\
\hline \multicolumn{2}{|c|}{$\begin{array}{l}\text { "Unha ensinanza no meu paso polos } \\
\text { estudos" }\end{array}$} & $\begin{array}{l}\text { "Unha experiencia } \\
\text { inesquecible" }\end{array}$ & $\begin{array}{c}\text { "Aprender non só é } \\
\text { aburrirse" }\end{array}$ \\
\hline $\begin{array}{l}\text { "Divertido e } \\
\text { educativo" }\end{array}$ & $\begin{array}{l}\text { "Aprendizaxe } \\
\text { dinámico" }\end{array}$ & \multicolumn{2}{|c|}{ "Proxecto interesante, necesario e divertido" } \\
\hline \multicolumn{2}{|c|}{ "Cooperar e aprender todos xuntos" } & de todas as persoas" & o mundo!" \\
\hline \multicolumn{2}{|c|}{ "Un proxecto para cambiar as cousas" } & \multicolumn{2}{|c|}{ "Aprender e imaxinar o mundo perfecto" } \\
\hline
\end{tabular}

Con el objetivo de que los resultados que el impacto del proyecto trasciendan al grupo que participa en él, trabajamos en dos niveles: a nivel de centro, mediante la difusión de metodologías y resultados entre el profesorado no implicado directamente en el proyecto y con la realización de un mural y de una presentación pública ante sus compañeras y compañeros de centro por parte del grupo participante; y a nivel de la sociedad gallega, a través de acciones de sensibilización y comunicación integradas, especialmente, en una sesión de trabajo especial, un encuentro intercentros que realizamos en primavera y en el que todo el alumnado participante se conoce, intercambia experiencias y conocimientos y trabaja de manera colaborativa en la elaboración de materiales de comunicación y sensibilización sobre una temática concreta, como las noticias falsas y su especial impacto en la visión que nuestra sociedad tiene de los movimientos migratorios. Este encuentro es recogido, cada año, por la televisión gallega y por los principales diarios y las principales emisoras de radio de Galicia, que entrevistan tanto a las y los docentes como a las y los alumnos que participan en el EPDLAB, completando así también sus procesos de empoderamiento mediático.

Al mismo tiempo, cada año ofrecemos una formación para el profesorado de otros centros, en colaboración con la Consejería de Educación de la Xunta de Galicia, con el objetivo de ampliar el impacto del proyecto más allá de los cinco institutos participantes. En este sentido, hemos documentado casos en los que tanto el profesorado que participa en la formación específica como el que forma parte del EPDLAB implementa algunas de las actividades del proyecto, aplicando incluso estas metodologías y herramientas a la enseñanza de otras materias.

\section{CONCLUSIONES}

EPDLAB es un proyecto de innovación educativa que, cada curso, acompaña a más de un ciento de alumnos y alumnas gallegas en el aprendizaje de las culturas de otros países, así como a ser críticos con la información que recibimos y a detectar noticias falsas y contenidos racistas o machistas en los medios, a aprender a utilizar el móvil "para algo más", a ejercer un consumo responsable, a colaborar con ONGDs o a deshacerse de los prejuicios. Todos estos resultados, así expresados, han salido de una de las evaluaciones colectivas en las que el alumnado identifica qué ha aprendido, valora los trabajos publicados en la web epdlab.gal y analiza qué impacto ha tenido el proyecto en ellas y ellos como personas. Por ello, podemos concluir que EPDLAB trabaja competencias $y$ contenidos que les resultan útiles a las y los estudiantes en su vida académica y personal, así como en su formación como ciudadanía activa y responsable, y que el propio alumnado es consciente de ello.

Más allá de esto, EPDLAB capacita al alumnado en el uso de herramientas sencillas y gratuitas al alcance de cualquiera, por lo que pueden continuar usándolas de forma autónoma una vez finalizada cada edición, cuestión que ha quedado probada desde la primera fase de este proyecto.

La relación con el profesorado es significativa, continua y muy satisfactoria. Hay centros que permanecen en el proyecto desde su inicio y todos manifiestan su interés para seguir participando cada año. Y, cuando el proyecto ha cambiado de centro, la vinculación con las y los docentes se ha mantenido, ya que siguen reutilizando herramientas y talleres a partir de la guía didáctica. Sabemos que muchas profesoras y profesores aplican esto a su trabajo en tutorías, educación en valores y a otras asignaturas, tanto de ámbito científico como de humanidades. La viabilidad (y la replicabilidad) del proyecto son patentes gracias al compromiso de los equipos docentes, que se implican cada día en el mismo y de los que nos consta su buena acogida y satisfacción con la metodología, contenidos $\mathrm{y}$ equipo técnico. Son su voluntad de formar parte de esta experiencia año tras año y su colaboración en todas las actividades las que hacen que este proyecto tenga futuro.

Cada dos años, se publica una guía didáctica bajo licencia CC-BY-SA, en la que incluimos todos los recursos empleados, materiales, objetivos, tiempos... Las estadísticas de descarga indican que esta guía es empleada por diferentes docentes para realizar todo el itinerario completo, o solo una parte, o incluso adaptar parte de los contenidos. Esto se refuerza también con la formación anual intensiva para al profesorado, que está 
homologada por la Consejería de Educación de la Xunta de Galicia y a la que acuden, principalmente, docentes recomendados tanto por el profesorado participante.

Los medios de comunicación han dado cobertura a EPDLAB en todas sus ediciones y han permitido que otros equipos docentes o de educación no formal manifiesten su interés en esta propuesta, llevando a nuestra organización a realizar talleres de forma independiente pero tangencial con la propia naturaleza del proyecto. Además, EPDLAB ha sido expuesto en varias ocasiones en la Facultad de Ciencias de la Educación de las universidades de Pontevedra y A Coruña, como contenido dentro de acciones de innovación educativa, y ha sido analizado como referencia en varias publicaciones, entre las que destaca la edición de la Revista Convives sobre Ciudadanía Digital.

Desde el equipo fundador del proyecto, consideramos fundamental de cara al futuro seguir trabajando en la difusión académica y profesional de EPDLAB, con el objetivo de conocer proyectos similares (lo que hasta el momento nos ha resultado imposible) y establecer sinergias que permitan replicarlo íntegramente o en parte en otros lugares de la geografía española.

\section{REFERENCIAS}

Adell, Jordi y Castañeda, Linda (2010), “Los Entornos Personales de Aprendizaje (PLEs): una nueva manera de entender el aprendizaje", En Roig Vila, R. \& Fiorucci, M. (Eds.) Claves para la investigación en innovación y calidad educativas. La integración de las Tecnologías de la Información y la Comunicación y la Interculturalidad en las aulas. Alcoy: Marfil - Roma TRE Universita degli studi:https://digitum.um.es/jspui/bitstream/10201/17247/ 1/Adell\&Casta $\%$ C3\%B1eda_2010.pdf

Álvarez, David, (2013), Capítulo 11: "Entornos personales de aprendizaje: del aprendizaje autónomo a la educación edupunk”. En Bergmann, Juliana y Grané, Mariona. La universidad en la nube. A universidade na nuvem (páginas 239-262) Barcelona: LMI.Col·lecció Transmedia XXI. Laboratori de Mitjans Interactius. Universitat de Barcelona. Barcelona. http://www.edutec.es/sites/default/files/publicaciones/6_ universidadnube.pdf

Boud, David (2002), "What is Peer Learning and why is it important?, Making the move to peer learning, in Peer Learning" en Higher Education: Learning From \& With Each Other, Boud David, Cohen Ruth \& Sampson, Jane:
https://web.stanford.edu/dept/CTL/Tomprof/postings/41 8.html

Embed.at. Contenidos del taller de micronarrativas digitales. Recuperado de: http://embed.at/contenidos-del-taller-demicronarrativas-audiovisuales/

EDPDLAB (2018). Guía Didáctica curso 17-18. Recuperado de: https://epdlab.gal/guia-didactica/

Freinet, Célestin (1997). Los métodos naturales I. El aprendizaje de la lengua. Fontanella.

Freire, Paulo (2012) Pedagogía del oprimido. Siglo XXI

Jenkins, Henry (2006). Convergence Culture: Where Old and New Media Collide

Kaplún, Mario (1998). Una pedagogía de la comunicación. Madrid: Editorial de la Torre

Ley Orgánica 8/2013, de 9 de diciembre, para la mejora de la calidad educativa. Recuperada de:

https://www.boe.es/buscar/act.php?id=BOE-A-2013-

12886. Competencias clave:

https://www.educacionyfp.gob.es/educacion/mc/lomce/el -curriculo/curriculo-primaria-eso-

bachillerato/competencias-clave/competenciasclave/digital.html

Objetivos de Desarrollo Sostenible (ODS) de la Organización de Naciones Unidas (ONU): https://www.un.org/sustainabledevelopment/es/

Pariser, Eli (2017). El filtro burbuja: como la web decide lo que leemos y lo que pensamos. Taurus: Madrid. https://www.youtube.com/watch?v=D6QMd9UbW2Q

Rowan, Jaron (2015). Memes. Inteligencia idiota, política rara y folclore digital.

https://www.academia.edu/33914729/Memes_Inteligenci a_idiota_pol\%C3\%ADtica_rara_y_folclore_digital

Vygotsky, Lev (2010) Pensamiento y lenguaje. Paidós: Barcelona.

Yáñez, M., Tello, L. y G. Gil, Felipe (2015) “Micronarrativas audiovisuales online", Espacio-Red de Prácticas y Culturas Digitales de la Universidad Internacional de Andalucía (UNIA) http://embed.at/contenidos-del-tallerde-micronarrativas-audiovisuales/

Zemos98 (2009) La Educación Expandida, recuperado de http://www.zemos98.org/descargas/educacion_expandid a-ZEMOS98.pdf 